\title{
Energy and energy spread measurements of an electron beam by Compton scattering method
}

\author{
C. Sun, ${ }^{1,2,3, *}$ J. Li, ${ }^{1,2,3}$ G. Rusev, ${ }^{1,3}$ A. P. Tonchev, ${ }^{1,3}$ and Y. K. Wu ${ }^{1,2,3}$ \\ ${ }^{1}$ Department of Physics, Duke University, Durham, North Carolina 27708-0305, USA \\ ${ }^{2}$ Duke Free Electron Laser Laboratory, Duke University, Durham, North Carolina 27708-0319, USA \\ ${ }^{3}$ Triangle Universities Nuclear Laboratory, Durham, North Carolina 27708-0308, USA
}

(Received 26 March 2009; published 18 June 2009)

\begin{abstract}
A gamma-ray beam produced by Compton scattering of a laser beam with an electron beam can be used to measure the electron beam parameters. In several published works, a simple fitting model has been applied to determine the electron beam energy and energy spread without considering the gamma beam collimation and electron beam emittance effects. This fitting model is rederived in this work, and the underlying assumptions and resultant limitations are discussed. To overcome these limitations, a new fitting model is proposed, which takes into account the collimation and emittance effects. Using the new model and a gamma-ray beam produced at the high intensity $\gamma$-ray sources facility at Duke University, we have successfully determined the electron beam energy with a relative uncertainty of about $3 \times 10^{-5}$ around $460 \mathrm{MeV}$ as well as the electron beam energy spread. We also experimentally demonstrated for the first time that a small relative energy change (about $4 \times 10^{-5}$ ) of the electron beam by varying the storage ring dipole field can be directly detected using the Compton scattering technique.
\end{abstract}

DOI: 10.1103/PhysRevSTAB.12.062801

PACS numbers: 13.60.Fz, 41.75.Ht, 29.20.D-, 29.30.Kv

\section{INTRODUCTION}

The energy of an electron beam in a storage ring can be measured with a relative uncertainty of few $10^{-3}$ from the integrated dipole field around the ring. However, a more accurate determination of the beam energy is required for mass measurements of meson particles produced in electron-position colliders [1,2], and the spectral-flux calculations of synchrotron radiation sources used for detector calibrations [3-5]. Two different methods, resonant spin depolarization [1,2,6] and Compton scattering [3,4,7-11], have been successfully applied to accurately determine the electron beam energy at some facilities. The method of resonant spin depolarization is based upon the measurement of the spin procession frequency of electrons in a guiding magnetic field. This method requires the ability to produce a polarized beam and the means to depolarize the beam. Using this method, the electron beam energy can be determined with a relative uncertainty on the order of $10^{-5}$. The application of this method is limited to high energy storage rings (typically above $1 \mathrm{GeV}$ ) in which the polarization of the electron beam can be built up within a reasonable amount of time. Compared to the resonant spin depolarization method, the Compton scattering method does not require a polarized beam, and is based upon the energy measurement of the Compton gamma beam. This method can be used for storage rings with a wide range of energies from a few hundred $\mathrm{MeV}$ to a few $\mathrm{GeV}$. The relative uncertainty of this method is usually on the order of $10^{-4}$.

\footnotetext{
*suncc@fel.duke.edu
}

In this paper, we focus on the Compton scattering method. The critical step is to find an accurate fitting model to describe the high energy edge of the measured gamma beam spectrum. In several published works $[3,4,9]$, the gamma beam spectrum edge was simply expressed as a convolution between a modified step function and a Gaussian function. The influences of the gamma beam collimation as well as the electron beam emittance on the gamma beam spectrum were not taken into account. However, under many circumstances, for example, the gamma-ray beam is tightly collimated, the gamma beam collimation and electron beam emittance could have significant impacts on the accuracy of the electron beam energy measurement. To overcome this problem, we have developed a new fitting model which can describe the gamma beam spectrum in detail, taking into account the collimation and emittance effects. Using this model, we have accurately measured the energy of the electron beam in the Duke storage ring.

Several published works $[4,9]$ also reported that the relative uncertainties of a few $10^{-5}$ had been achieved for the electron beam energy measurements. However, all these measurements were carried out for a high energy storage ring above $1 \mathrm{GeV}$. We experimentally demonstrate that this level of accuracy of a few $10^{-5}$ can also be achieved for a low energy storage ring around a few hundred $\mathrm{MeV}$ using well-calibrated detectors.

\section{ENERGY OF COMPTON SCATTERED PHOTON}

The kinematic process of the Compton scattering of a photon with an electron is illustrated in Fig. 1. According to the principle of four-momentum conservation, the en- 


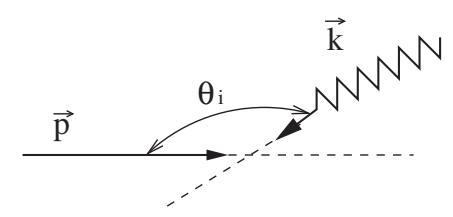

(a) Before scattering

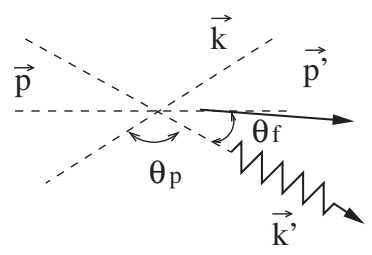

(b) After scattering
FIG. 1. Diagram of Compton scattering process. The vectors $\vec{p}$ and $\vec{k}$ represent the momenta of the electron and photon before the scattering, respectively. The vectors $\vec{p}^{\prime}$ and $\vec{k}^{\prime}$ represent the momenta of the electron and photon after the scattering, respectively. $\theta_{i}$ is the angle between $\vec{p}$ and $\vec{k}, \theta_{p}$ the angle between $\vec{k}$ and $\vec{k}^{\prime}$, and $\theta_{f}$ the angle between $\vec{p}$ and $\vec{k}^{\prime}$.

ergy of scattered photon is given by the following expression $[12,13]$ :

$$
E_{\gamma}=\frac{E_{p}\left(1-\beta \cos \theta_{i}\right)}{1-\beta \cos \theta_{f}+\frac{E_{p}}{E_{e}}\left(1-\cos \theta_{p}\right)},
$$

where $E_{p}$ and $E_{e}$ are the energy of photon and electron before scattering, respectively; $\beta=v / c$ is the velocity of the electron scaled by the speed of light $c ; \theta_{i}$ is the angle between the momenta of the incident electron and photon as shown in Fig. $1 ; \theta_{f}$ is the angle between the momenta of the scattered photon and incident electron; $\theta_{p}$ is the angle between the momenta of the scattered and incident photons.

For head-on collisions $\left(\theta_{i}=\pi, \theta_{p}=\pi-\theta_{f}\right)$ of relativistic electrons $(\beta \approx 1)$ with laser photons, the scattered photons are concentrated around the incident electron direction within a cone of an opening angle of $1 / \gamma$, where $\gamma$ is the Lorentz factor, $\gamma=1 / \sqrt{1-\beta^{2}}$. Thus, the energy of the scattered photon can be expressed approximately as $[8,14]$

$$
E_{\gamma} \approx \frac{4 \gamma^{2} E_{p}}{1+\gamma^{2} \theta_{f}^{2}+4 \gamma^{2} E_{p} / E_{e}} .
$$

When $\theta_{f}=0$ (backscattering), the scattered photon will reach the maximum energy

$$
E_{\gamma}^{\max }=E_{\gamma}^{H}\left(E_{e}, E_{p}\right)=\frac{4 \gamma^{2} E_{p}}{1+4 \gamma^{2} E_{p} / E_{e}},
$$

where $E_{\gamma}^{H}\left(E_{e}, E_{p}\right)$ is a notation to be used in the next section to represent the highest possible scattered photon energy by colliding an electron of energy $E_{e}$ and a laser photon of energy $E_{p}$.

Neglecting the recoil term $\left(4 \gamma^{2} E_{p} / E_{e} \ll 1\right)$, Eq. (3) can be further simplified to

$$
E_{\gamma}^{\max } \approx 4 \gamma^{2} E_{p}
$$

Thus, the incident photon energy $E_{p}$ is approximately enhanced by a factor of $4 \gamma^{2}$ after the backscattering. Therefore, the Compton scattering of a laser photon with a relativistic electron can produce high energy photons, i.e., gamma-ray photons. The rms relative uncertainty of $E_{\gamma}^{\max }$ is determined by the uncertainties of the parameters entering Eq. (4) [15], i.e.,

$$
\frac{\sigma_{E_{\gamma}^{\max }}}{E_{\gamma}^{\max }} \approx \sqrt{\left(2 \frac{\sigma_{E_{e}}}{E_{e}}\right)^{2}+\left(\frac{\sigma_{E_{p}}}{E_{p}}\right)^{2}}
$$

where $\sigma_{E_{e}}$ and $\sigma_{E_{p}}$ represent the rms uncertainties of the electron and laser photon energy, respectively.

\section{SPECTRUM OF COMPTON SCATTERED GAMMA-RAY BEAM}

A gamma-ray beam produced by Compton scattering of a laser beam with a relativistic electron beam has been used to measure the electron beam energy and energy spread in several published works $[3,4,9]$. The electron beam energy and energy spread were determined by fitting the high energy edge of the measured gamma beam spectrum using a simple fitting model introduced in paper [3]. However, the underlying assumptions and resultant limitations of this model have not been fully discussed. In this section, we will rederive the result presented in paper [3], and provide detailed discussions about the assumptions and limitations. To overcome these limitations, we will propose a new fitting model for the accurate determination of the electron beam energy and energy spread.

\section{A. A simple fitting model}

For a head-on collision of a monoenergetic pointlike electron and laser beams, the highest energy of the scattered gamma-ray photons can be directly determined using Eq. (3). This equation also allows determination of the electron beam energy $E_{e}$ if the laser photon energy $E_{p}$ and the highest gamma photon energy $E_{\gamma}^{H}$ are known. Usually, the laser wavelength can be accurately measured by a spectrometer. Therefore, the critical step using Compton scattering to measure the electron beam energy is to accurately determine $E_{\gamma}^{H}$ from the measured energy spectrum of the gamma-ray beam. Theoretically, the energy spectrum of a gamma-ray beam produced by a headon collision of a monoenergetic pointlike electron and laser beams can be approximately described by the formula $[10,13,16]$

$$
\begin{aligned}
\frac{d \sigma}{d E_{\gamma}}= & \frac{\pi r_{e}^{2}}{2 \gamma^{2} E_{p}}\left[\frac{E_{e}^{2}}{4 \gamma^{4} E_{p}^{2}}\left(\frac{E_{\gamma}}{E_{e}-E_{\gamma}}\right)^{2}-\frac{E_{e}}{\gamma^{2} E_{p}} \frac{E_{\gamma}}{E_{e}-E_{\gamma}}\right. \\
& \left.+\frac{E_{e}}{E_{e}-E_{\gamma}}+\frac{E_{e}-E_{\gamma}}{E_{e}}\right] \text { for } E_{\gamma} \leq E_{\gamma}^{H}
\end{aligned}
$$

where $r_{e}$ is the classical electron radius. Neglecting the recoil effect, this formula can be further simplified to 


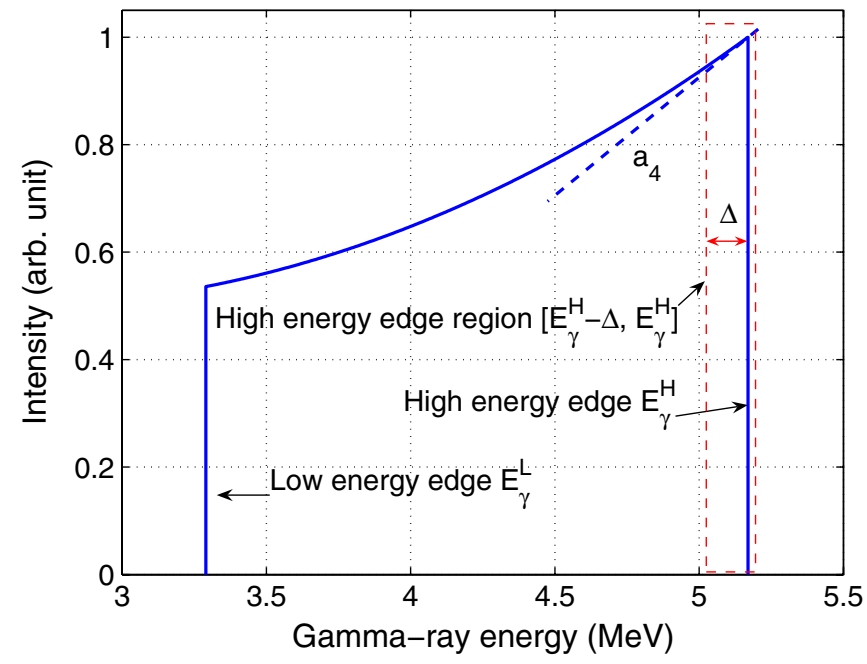

FIG. 2. (Color) The energy spectrum of a Compton gamma-ray beam produced by the head-on collision of a $466 \mathrm{MeV}$ pointlike electron beam with a $789 \mathrm{~nm}$ pointlike laser beam. A collimation aperture with radius of $50 \mathrm{~mm}$ is placed $60 \mathrm{~m}$ downstream from the collision point. The low energy edge $E_{\gamma}^{L}$ is determined by the collimation acceptance, while the high energy edge $E_{\gamma}^{H}$ is determined by the electron and laser photon energy. The slope of the spectrum at the high energy edge is denoted as $a_{4}$.

$$
\frac{d \sigma}{d E_{\gamma}} \approx \frac{4 \pi r_{e}^{2}}{E_{\gamma}^{H}}\left[1-2 \frac{E_{\gamma}}{E_{\gamma}^{H}}+2\left(\frac{E_{\gamma}}{E_{\gamma}^{H}}\right)^{2}\right] \text { for } E_{\gamma} \leq E_{\gamma}^{H} .
$$

An example spectrum calculated using this formula is shown in Fig. 2. Clearly, the high energy edge of the spectrum is a step function from which $E_{\gamma}^{H}$ can be determined.

In practice, the electron and laser beams have a finite energy and angular distributions. As a result, the gamma beam spectrum cannot be directly described by Eq. (7). However, it can be calculated by a weighted integral of a series of spectra described by Eq. (7), where the weighting function is given by the actual energy and angular distributions of the electron and laser beams. In the following derivation, we consider a head-on collision of a pointlike electron and laser beams, and with a fixed laser photon energy $E_{p}$. Further, we assume that the energy distribution of the electron beam is a Gaussian function with a centroid energy of $E_{e 0}$. Thus, the weighting function for the spectrum [Eq. (7)] which has the highest possible energy $E_{\gamma}^{H}$ can be expressed as

$$
g\left(E_{\gamma}^{H}\right)=\frac{1}{\sqrt{2 \pi} a_{2}} \exp \left[-\frac{\left(E_{\gamma}^{H}-a_{1}\right)^{2}}{2 a_{2}^{2}}\right]
$$

where $a_{1}=E_{\gamma}^{H}\left(E_{e 0}, E_{p}\right)$, representing the highest possible gamma photon energy associated with the electron energy $E_{e 0}$ according to Eq. (3); and $a_{2}$ represents the rms energy spread of the gamma beam caused by the rms energy spread of the electron beam, i.e., $a_{2} / a_{1} \approx 2 \sigma_{E_{e}} / E_{e 0}$ [see Eq. (5) with $\sigma_{E_{p}}=0$ ].

To focus on the high energy edge region $\left(E_{\gamma}^{H}-\Delta, E_{\gamma}^{H}\right)$ of the spectrum shown in Fig. 2, and assuming $\Delta / E_{\gamma}^{H} \ll 1$, the energy spectrum described by Eq. (7) can be simplified to

$$
\begin{aligned}
& \frac{d \sigma}{d E_{\gamma}} \approx \frac{4 \pi r_{e}^{2}}{E_{\gamma}^{H}}\left[1+\frac{2}{E_{\gamma}^{H}}\left(E_{\gamma}-E_{\gamma}^{H}\right)\right] \\
& \quad \text { for } E_{\gamma}^{H}-\Delta \leq E_{\gamma} \leq E_{\gamma}^{H} .
\end{aligned}
$$

For simplicity, we can rewrite the above equation to a modified step function

$$
\begin{gathered}
h\left(E_{\gamma}, E_{\gamma}^{H}\right)=a_{3}\left[1+a_{4}\left(E_{\gamma}-E_{\gamma}^{H}\right)\right] \\
\text { for } E_{\gamma}^{H}-\Delta \leq E_{\gamma} \leq E_{\gamma}^{H},
\end{gathered}
$$

where the parameters $a_{3}=4 \pi r_{e}^{2} / E_{\gamma}^{H}$ and $a_{4}=2 / E_{\gamma}^{H}$ represent the intensity and slope of the spectrum, respectively.

Integrating the modified step function $h\left(E_{\gamma}, E_{\gamma}^{H}\right)$ weighted by the function $g\left(E_{\gamma}^{H}\right)$, and using the complementary error function

$$
\operatorname{erfc}(x)=\frac{2}{\sqrt{\pi}} \int_{x}^{\infty} \exp \left(-t^{2}\right) d t
$$

we can obtain an approximate description of the collective gamma beam spectrum at the high energy edge, which reproduces the result presented in paper [3],

$$
\begin{aligned}
f\left(E_{\gamma}, a 1, \ldots, a_{5}\right) & =\int_{0}^{\infty} h\left(E_{\gamma}, E_{\gamma}^{H}\right) g\left(E_{\gamma}^{H}\right) d E_{\gamma}^{H}+a_{5} \approx \frac{a_{3}}{\sqrt{2 \pi} a_{2}} \int_{E_{\gamma}}^{\infty}\left[1+a_{4}\left(E_{\gamma}-E_{\gamma}^{H}\right)\right] \times \exp \left(-\frac{\left(E_{\gamma}^{H}-a_{1}\right)^{2}}{2 a_{2}^{2}}\right) d E_{\gamma}^{H}+a_{5} \\
& =a_{3}\left\{\frac{1}{2}\left[1+a_{4}\left(E_{\gamma}-a_{1}\right)\right] \times \operatorname{erfc}\left(\frac{E_{\gamma}-a_{1}}{\sqrt{2} a_{2}}\right)-\frac{a_{2} a_{4}}{\sqrt{2 \pi}} \times \exp \left(-\frac{\left(E_{\gamma}-a_{1}\right)^{2}}{2 a_{2}^{2}}\right)\right\}+a_{5},
\end{aligned}
$$

where the parameter $a_{5}$ represents the spectrum offset. This equation can be used to fit the high energy edge of the Compton gamma-ray beam spectrum to determine the electron beam energy and energy spread using $a_{1}, \ldots, a_{5}$ as fitting parameters.
Note that in principle, $a_{3}$ and $a_{4}$ in Eq. (12) depend on the integration variable $E_{\gamma}^{H}$. However, they are assumed to be constant during the integration in order to derive the exact form of Eq. (12). This frees up $a_{3}$ and $a_{4}$ as two independent fitting parameters. While this improves the 
fitting, the fitting result of $a_{4}$ could be nonphysical in some circumstances. When the fit value of $a_{4}$ is significantly different from its physical value of $2 / E_{\gamma}^{H}$, Eq. (12) is found to be inaccurate in describing the high energy edge of the gamma beam spectrum. As shown in the following section, this limits use of Eq. (12) for the accurate determination of the electron beam energy and energy spread.

\section{B. Gamma beam collimation and electron beam emittance effects}

In practice, the Compton gamma-ray beam is first collimated by a round aperture (a lead collimator) and then measured by a gamma-ray detector. Thus, the gamma beam divergence after the collimation is given by

$$
\Delta \theta_{c}=\frac{R}{L},
$$

where $R$ is the radius of the collimation aperture, and $L$ is the distance between the collision point and the collimator. In addition to the contribution caused by the electron beam energy spread, the opening angle $\Delta \theta_{c}$ of the collimator also leads to a contribution to the energy spread of the gamma-ray beam.

For a head-on collision of a monoenergetic pointlike electron and laser beams, according to Eq. (2) the relative full-width energy spread $\Delta E_{\gamma} / E_{\gamma}$ of the gamma beam after the collimation is given by the expression

$$
\frac{\Delta E_{\gamma}}{E_{\gamma}^{H}} \equiv \frac{E_{\gamma}^{H}-E_{\gamma}^{L}}{E_{\gamma}^{H}} \approx \gamma^{2} \Delta \theta_{c}^{2},
$$

where $E_{\gamma}^{L}$ represents the minimum energy of the gamma photons accepted by the collimator, i.e., the low energy edge of the spectrum shown in Fig. 2. $E_{\gamma}^{H}$ represents the high energy edge of the spectrum, and is only determined by electron and laser photon energies according to Eq. (3).

However, if the electron beam has a finite energy spread, the high energy edge of the gamma beam spectrum could be influenced by the collimation aperture. Especially, when the gamma beam energy spread due to the collimation is smaller than or comparable to that due to the electron beam energy spread, i.e., $\gamma^{2} \Delta \theta_{c}^{2} \leq 2 \sigma_{E_{e}} / E_{e}$, the collimation effect will start to alter the high energy edge of the spectrum, resulting in a shift of the spectrum peak toward the higher energy. In this case, the electron beam emittance will also play a role in shaping the gamma beam spectrum.

Since Eq. (12) does not take into account the gamma beam collimation and electron beam emittance effects, its application to determine the electron beam energy becomes less accurate when these effects are significant.

\section{A comprehensive fitting model}

In order to include the gamma beam collimation and electron beam emittance effects in the calculation of the gamma beam spectrum, we must start with a more fundamental formula of the angular and energy distribution of the gamma photons produced by the collision of an electron and a laser bunches:

$$
\begin{aligned}
\frac{d^{2} N_{\gamma}}{d \Omega_{L} d E_{\gamma}}= & N_{e} N_{p} \int \frac{d^{2} \sigma}{d \Omega d E_{\gamma}} c\left(1-\beta \cos \theta_{i}\right) f_{e}\left(\mathbf{V}, \mathbf{p}_{e}, t\right) \\
& \times f_{p}(\mathbf{V}, \mathbf{k}, t) d \mathbf{p}_{e} d \mathbf{k} d \mathbf{V} d t
\end{aligned}
$$

where $d N_{\gamma}$ is the number of the gamma photons in an energy range of $E_{\gamma}$ to $E_{\gamma}+d E_{\gamma}$ and solid angle $d \Omega_{L}$ viewed in the laboratory frame; $N_{e}$ and $N_{p}$ are the total numbers of electrons and laser photons in their respective bunches; $d \sigma / d \Omega$ is the angular differential cross section for Compton scattering [13]; $f_{e}\left(\mathbf{V}, \mathbf{p}_{e}, t\right)$ and $f_{p}(\mathbf{V}, \mathbf{k}, t)$ are the phase space distribution functions of the electron and photon beams. The integration is computed for the entire collision time and volume as well as the momenta of electrons and laser photons via $\int \cdots d \mathbf{p}_{e} d \mathbf{k} d \mathbf{V} d t$.

Assuming Gaussian distributions of an unpolarized electron and laser beams, neglecting the vertical emittance of the electron beam and the energy spread of the laser beam, and further assuming head-on collision happening at the waist of the laser beam, the energy spectrum of the collimated gamma beam can be obtained by partially integrating Eq. (15) [12,17,18],

$$
\begin{aligned}
\frac{d N_{\gamma}}{d E_{\gamma}} \approx & \frac{r_{e}^{2} L^{2} N_{e} N_{p}}{2 \pi^{2} \hbar c \beta_{0} \sqrt{\zeta_{x}} \sigma_{\gamma} \sigma_{\theta x}} \int_{-y_{o}}^{y_{o}} \int_{-x_{o}}^{x_{o}} \int_{-\theta_{x \max }}^{\theta_{x \max }}\left(\frac{\bar{\gamma}}{1+2 \bar{\gamma} a}\right) \times\left\{\frac{1}{4}\left[\frac{4 \bar{\gamma}^{2} E_{p}}{E_{\gamma}\left(1+\bar{\gamma}^{2} \theta_{f}^{2}\right)}+\frac{E_{\gamma}\left(1+\bar{\gamma}^{2} \theta_{f}^{2}\right)}{4 \bar{\gamma}^{2} E_{p}}\right]-\frac{\bar{\gamma}^{2} \theta_{f}^{2}}{\left(1+\bar{\gamma}^{2} \theta_{f}^{2}\right)^{2}}\right\} \\
& \times \exp \left(-\frac{\left(\theta_{x}-x_{c} / L\right)^{2}}{2 \sigma_{\theta_{x}}^{2}}-\frac{\left(\bar{\gamma}-\gamma_{0}\right)^{2}}{2 \sigma_{\gamma}^{2}}\right) d \theta_{x} d x_{c} d y_{c},
\end{aligned}
$$

where 


$$
\begin{gathered}
\bar{\gamma}=\frac{2 E_{\gamma} a}{4 E_{p}-E_{\gamma} \theta_{f}^{2}}\left(1+\sqrt{\left.1+\frac{4 E_{p}-E_{\gamma} \theta_{f}^{2}}{4 a^{2} E_{\gamma}}\right)} ; \quad a=\frac{E_{p}}{m c^{2}} ; \quad \theta_{f}^{2}=\theta_{x}^{2}+\left(\frac{y_{c}}{L}\right)^{2} ; \quad \sigma_{\theta x}=\sqrt{\frac{\varepsilon_{x} \xi_{x}}{\beta_{x} \zeta_{x}}}\right. \\
\xi_{x}=1+\left(\frac{\beta_{x}}{L}\right)^{2}+\frac{2 k \beta_{x} \varepsilon_{x}}{\beta_{0}} ; \quad \zeta_{x}=1+\frac{2 k \beta_{x} \varepsilon_{x}}{\beta_{0}} ; \quad \theta_{x \max }=\sqrt{\frac{4 E_{p}}{E_{\gamma}}-\left(\frac{y_{c}}{L}\right)^{2}}
\end{gathered}
$$

$\hbar$ is the reduced Planck constant; $c$ is the speed of light; $\varepsilon_{x}$ and $\beta_{x}$ are the emittance and the beta function of the electron beam in the horizontal direction, respectively; $\gamma_{0}=E_{e} / m c^{2}$ and $\sigma_{\gamma}=\sigma_{E_{e}} / m c^{2}$ represent the electron beam energy and energy spread normalized by $m c^{2}$, respectively; $\beta_{0}$ and $k=E_{p} /(\hbar c)$ are the Rayleigh range and wave number of the laser beam, respectively; $L$ is the distance between the collision point and the collimator; $x_{o}$ and $y_{o}$ are half widths of horizontal and vertical apertures, and for a circular aperture, the radius of the aperture is given by $R=\sqrt{x_{o}^{2}+y_{o}^{2}}$. Based upon the assumption of far field collimation $(L \gg R)$, the solid angle $d \Omega_{L}$ in Eq. (15) has been replaced by $d x_{c} d y_{c} / L^{2}$, where $x_{c}$ and $y_{c}$ are integration variables ranging from $-x_{o}$ to $x_{o}$ and from $-y_{o}$ to $y_{o}$, respectively.

Equation (16) has been derived under assumptions of an unpolarized Gaussian laser beam with a zero energy spread scattering with an unpolarized Gaussian electron beam. In order to simplify the integrations, the vertical emittance of the electron beam has also been neglected in Eq. (16). For many storage rings, this is a good approximation because the vertical emittance is much smaller than the horizontal one. When these assumptions are no longer valid, Eq. (16) can be easily extended by adding additional integrals to include other beam distribution effects [18], such as the vertical divergence of the electron beam and the energy spread of the laser beam. Equation (16) can also be extended to a polarized laser beam case by modifying the scattering cross section. In addition, the alignment of the collimator to the gamma beam can been easily introduced in Eq. (16) by changing the integration range for the variables $x_{c}$ and $y_{c}$.

\section{Energy spectrum of collimated Compton gamma-ray beam}

In order to use Eq. (16) to calculate the energy spectrum $d N_{\gamma} / d E_{\gamma}$ of a collimated Compton gamma-ray beam, the integrations with respect to $d x_{c}, d y_{c}, d \theta_{x}$ must be evaluated numerically. For this purpose, a numerical integration code has been developed [18]. The spectra calculated using this code are shown in Figs. 3 and 4.

Figure 3 illustrates the influence of the collimation aperture on the energy spectrum of the gamma beam. The spectra are calculated for collimators with varying aperture radius $R$. To minimize the emittance effect, a small electron beam emittance of $0.05 \mathrm{~nm}$ rad is used for the calculation. In order to compare the collimation effect
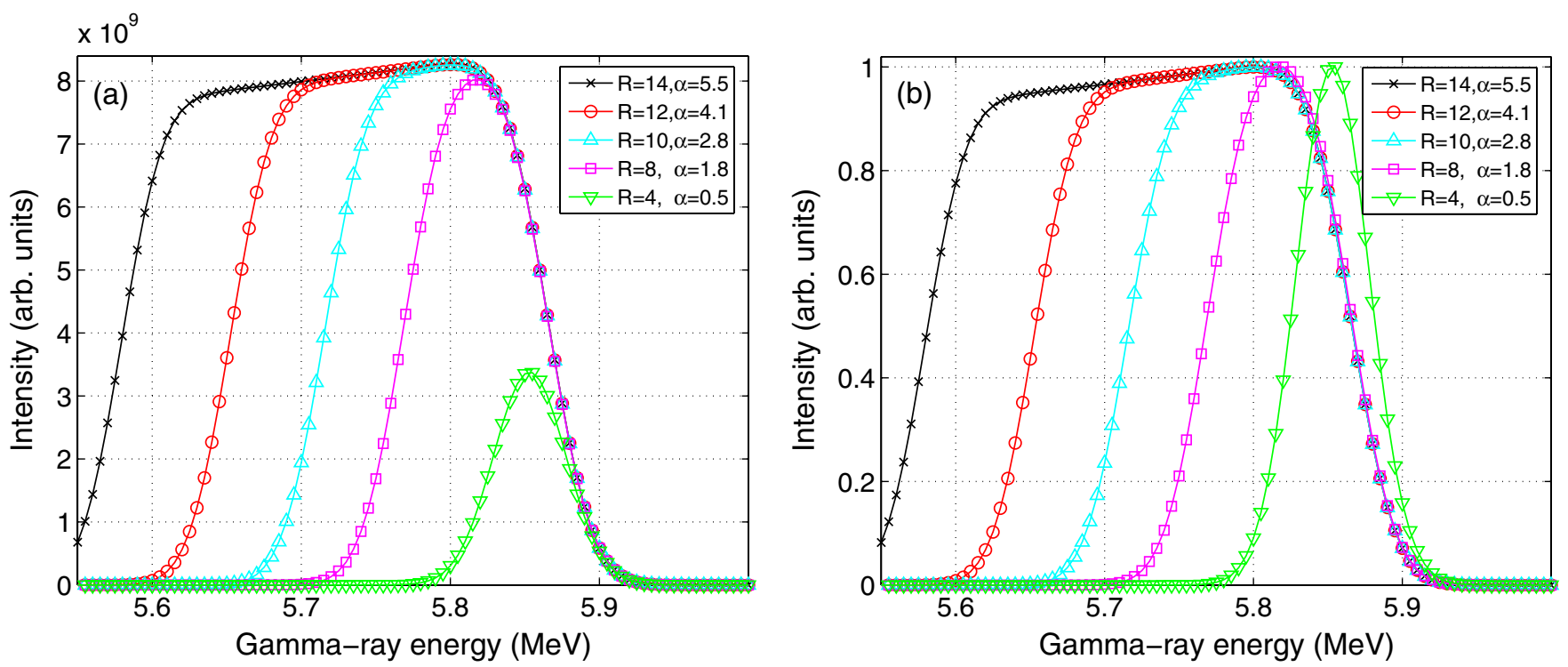

FIG. 3. (Color) Calculated energy spectra of gamma beams produced by Compton scattering of a $800 \mathrm{~nm}$ laser beam with a $500 \mathrm{MeV}$ electron beam for different radii of the collimation aperture. The aperture is placed $60 \mathrm{~m}$ downstream from the collision point, and its radius $R$ is varied from 14 to $4 \mathrm{~mm}$. $\alpha$ is defined in Eq. (17). The horizontal emittance and energy spread of the electron beam are fixed at $0.05 \mathrm{~nm}$ rad and $2 \times 10^{-3}$, respectively. (a) Spectra are normalized to the intensities of incident electron and laser beams. (b) Spectra are scaled to their respective peak values. 

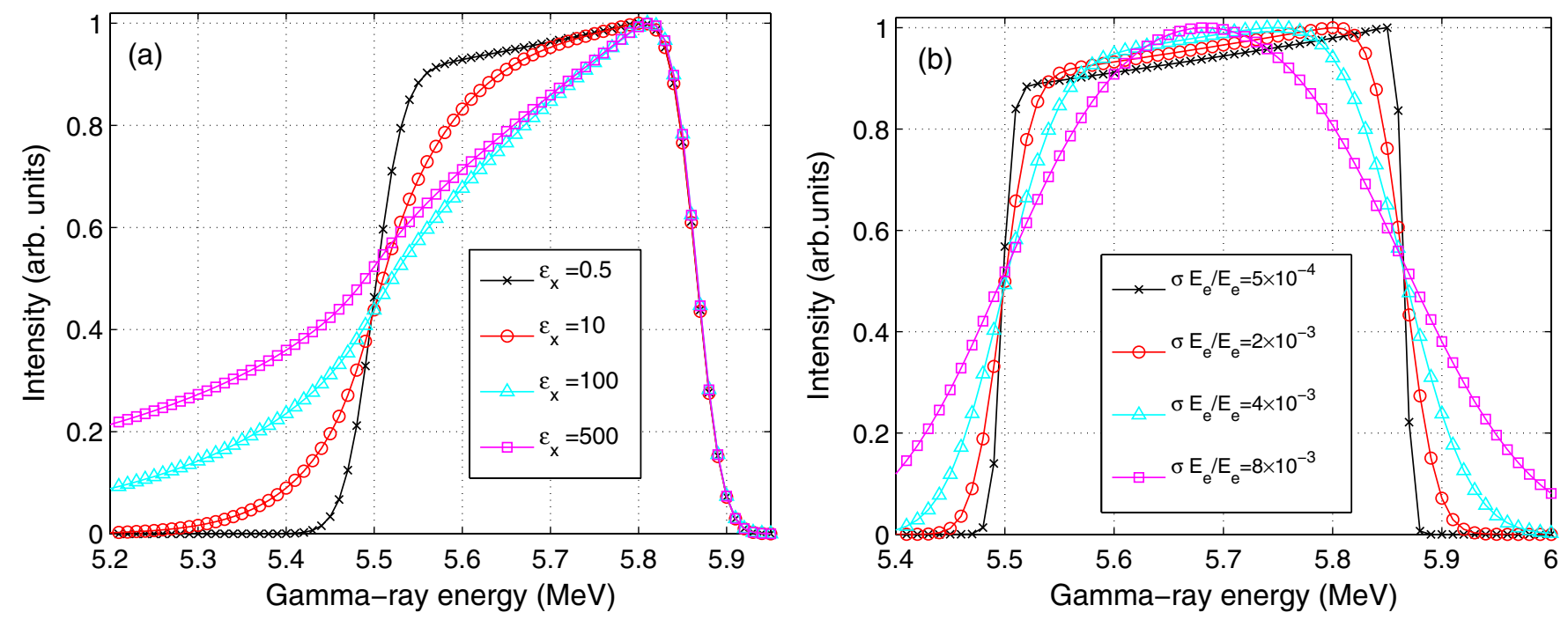

FIG. 4. (Color) Calculated energy spectra of gamma beams produced by Compton scattering of a $800 \mathrm{~nm}$ laser beam with a $500 \mathrm{MeV}$ electron beam for different horizontal emittances $\varepsilon_{x}$ and energy spread $\sigma_{E_{e}}$ of the electron beam. The gamma beam is collimated by an aperture with radius of $16 \mathrm{~mm}$ which is placed $60 \mathrm{~m}$ downstream from the collision point. The spectra are scaled to their respective peak values. (a) The horizontal emittance $\varepsilon_{x}$ of the electron beam is varied from 0.5 to $500 \mathrm{~nm}$ rad, while the relative energy spread is fixed at $2 \times 10^{-3}$. (b) The relative energy spread $\sigma_{E_{e}} / E_{e}$ is varied from $5 \times 10^{-4}$ to $8 \times 10^{-3}$, while the horizontal emittance $\varepsilon_{x}$ is fixed at $0.05 \mathrm{~nm}$ rad.

to the electron beam energy spread effect, a relative collimation factor $\alpha$ can be defined as

$$
\alpha=\frac{\gamma^{2} \Delta \theta_{c}^{2}}{2 \sqrt{2 \ln 2} \times\left(2 \sigma_{E_{e}} / E_{e}\right)},
$$

where $2 \sqrt{2 \ln 2}$ is the conversion factor between FWHM and the rms width. Figure 3(a) shows that the collimation cuts down the lower energy gamma beam intensity, and determines the low energy edge of the spectrum. For a large collimation aperture $(\alpha>3)$, the low and high energy edges of the spectrum are well separated, thus the high energy edge is not influenced by the collimation aperture. However, for a tight collimation $(\alpha<2)$, the low and high energy edges begin to join together, and the peak of the spectrum shifts toward the higher energy end as $\alpha$ is decreased. This is more clearly demonstrated in Fig. 3(b) in which gamma beam spectra are scaled to their peak values.

Figure 4(a) illustrates the influence of the electron beam emittance on the shape of the gamma beam spectrum. To minimize the collimation effect on the high energy edge of the gamma beam spectrum, a large collimation aperture $(\alpha \approx 7.2)$ is used in the calculation. The spectra shown in Fig. 4(a), scaled to their respective peak values, are calculated for electron beams with varying horizontal emittance $\varepsilon_{x}$. The figure shows that for a large collimation aperture an increased electron beam emittance spreads the low energy edge of the gamma spectrum, while leaving the higher energy side of the spectrum practically unchanged. For a tightly collimated gamma beam (not shown in the figure), the low and high energy edges of the spectrum join together. In this case, the electron beam emittance will begin to have an impact on the spectrum high energy edge.

Figure 4(b) illustrates the influence of the electron beam energy spread on the shape of gamma beam spectrum. To minimize the collimation and emittance effects on the gamma beam spectrum, a small electron beam emittance and large collimation aperture are used in the calculation. The spectra shown in Fig. 4(b), scaled to their respective peak values, are calculated for electron beams with varying energy spread $\sigma_{E_{e}}$. Clearly, unlike the electron beam emittance effect, a nonmonoenergetic electron beam spreads the Compton gamma rays in a wider energy range, smearing both the low and high energy edges of the gamma spectrum.

The gamma beam spectra can also be influenced by the alignment offset of the collimation aperture with respect to the gamma beam, which is illustrated in Fig. 5. When the misalignment offset is small compared to the collimation aperture size, it will not have a significant impact on the high energy edge of the gamma spectrum. In this case, the effect of a misaligned aperture on the gamma beam spectrum is similar to that of electron beam emittance.

In general, the high energy edge of a collimated Compton gamma beam spectrum is influenced by the energy spread and emittance of the electron beam as well as the aperture size and alignment of the collimation aperture. However, Eq. (12) only includes the effect of the electron beam energy spread, therefore is not adequate for the cases when other effects are important. In particular, 


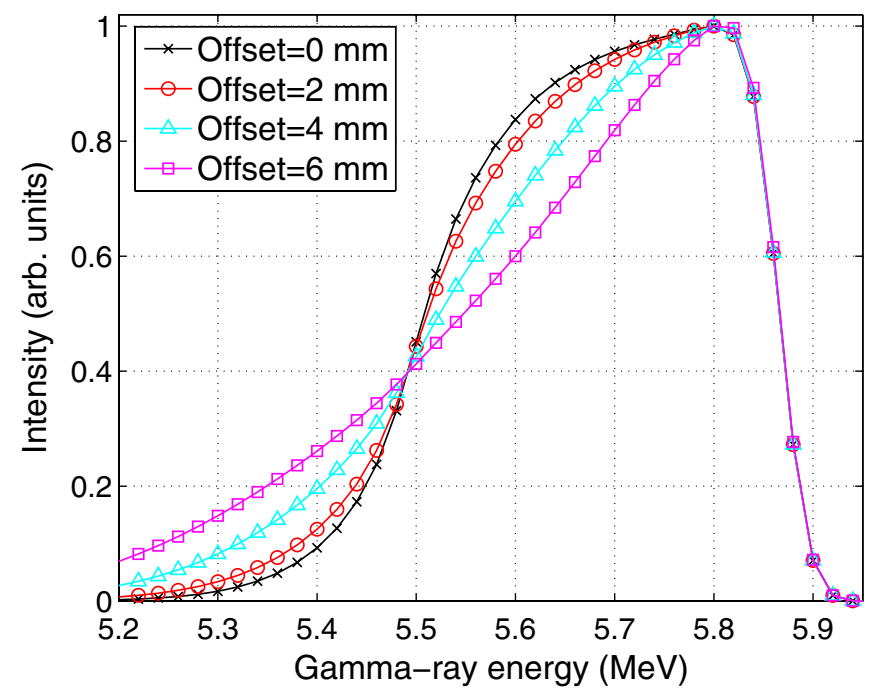

FIG. 5. (Color) Calculated energy spectra of gamma beams produced by Compton scattering of a $800 \mathrm{~nm}$ laser beam with a $500 \mathrm{MeV}$ electron beam for different alignment offsets of the collimator. The collimator with an aperture radius of $16 \mathrm{~mm}$ is placed $60 \mathrm{~m}$ downstream from the collision point. The electron beam energy spread and horizontal emittance are fixed to $2 \times$ $10^{-3}$ and $10 \mathrm{~nm}$ rad, respectively. The spectra are scaled to their respective peak values.

when the gamma-ray beam is tightly collimated (a small $\alpha$ ), the accurate determination of the electron beam energy will require a new fitting model such as Eq. (16).

\section{E. Validating fitting formulas}

A direct test of Eqs. (16) and (12) is to use them to fit electron beam energy for a few test spectra generated by a Monte Carlo simulation code such as CAIN 2.35 [19]. The same beam parameters as described in Fig. 3(a) were used in the simulations, but with an electron beam emittance of $10 \mathrm{~nm}$ rad and a varied radius of the collimation aperture from 2.0 to $18 \mathrm{~mm}$. As a result, the relative collimation factor $\alpha$ is varied from 0.10 to 9.1. The fitting results using both Eqs. (12) and (6) are summarized in Fig. 6.

It shows that regardless of the collimation aperture size Eq. (16) can always determine the electron beam energy correctly with a high accuracy of $2 \times 10^{-5}$ or better. However, to obtain the similar accuracy using Eq. (12) requires a relatively large collimation aperture $(\alpha>4)$. With a smaller aperture, the accuracy of Eq. (12) is significantly lower. For example, when $\alpha<1$, the accuracy is reduced to about $10^{-3}$. In this case, the Compton method of determining the electron beam energy using Eq. (12) has no significant advantage over the simpler method of using the integrated dipole magnetic field.

In the region of $1<\alpha<4$, Eq. (12) can still determine the electron beam energy with an accuracy of $10^{-4}$, a better result compared with the case of $\alpha<1$. This improvement is the result of using the coefficient $a_{4}$ in Eq. (12) as a

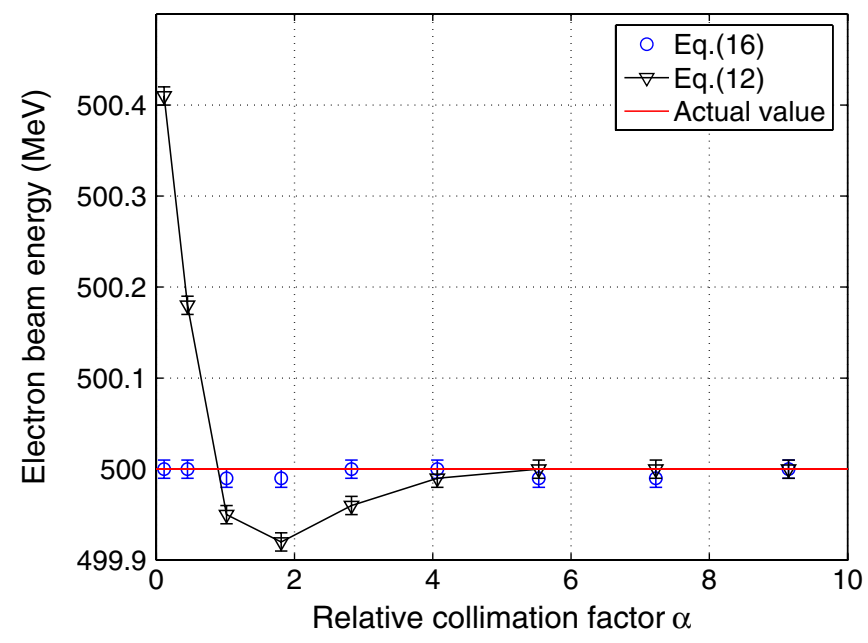

FIG. 6. (Color) The fit electron beam energy as a function of the relative collimation factor $\alpha$. Both Eq. (16) and Eq. (12) are used for the determination of the electron beam energy. The error bars represent fitting errors. The horizontal line represents the actual energy value of the electron beam used in producing simulated gamma beam spectra.

fitting parameter to take on different values for different collimation apertures. In this case, the fit value of $a_{4}$ ranges from 0.40 to $10 \mathrm{MeV}^{-1}$ as the collimation aperture radius is decreased from 12 to $6.0 \mathrm{~mm}$ or $\alpha$ from 4.0 to 1.0 . However, the physics value of $a_{4}$, which is given by $2 / E_{\gamma}^{H}$, should be independent of the collimation aperture size, and is approximately equal to $0.40 \mathrm{MeV}^{-1}$. This artificial increase of $a_{4}$ compensates the decrease of the intensity at the lower energy side of the spectrum as the collimation aperture size decreases. Although this is nonphysical, it can produce a better fit.

When $\alpha>4$, the high energy edge of the spectrum is only weakly affected by the collimation aperture. Thus, the fit values of $a_{4}$ have a weak dependence on the collimation aperture size, and become close to its physics value of about $0.40 \mathrm{MeV}^{-1}$. In this case, Eq. (12) can determine the electron beam energy with an accuracy similar to Eq. (16).

\section{MEASUREMENTS OF ELECTRON BEAM ENERGY AND ENERGY SPREAD}

\section{A. Experimental setup}

The high intensity $\gamma$-ray source $(\mathrm{HI} \gamma \mathrm{S})$ facility at Duke University has been recently upgraded to improve its performance [20,21]. The schematic layout of this facility is shown in Fig. 7. The gamma-ray beam at $\mathrm{HI} \gamma \mathrm{S}$ is generated by colliding a free-electron laser (FEL) beam inside the laser resonator with an electron beam in the storage ring. The electron beam is first generated and accelerated to $180 \mathrm{MeV}$ in a linear accelerator. The electron beam energy is then ramped up to a desired value in a booster synchrotron before injecting into the storage ring. The energy of 


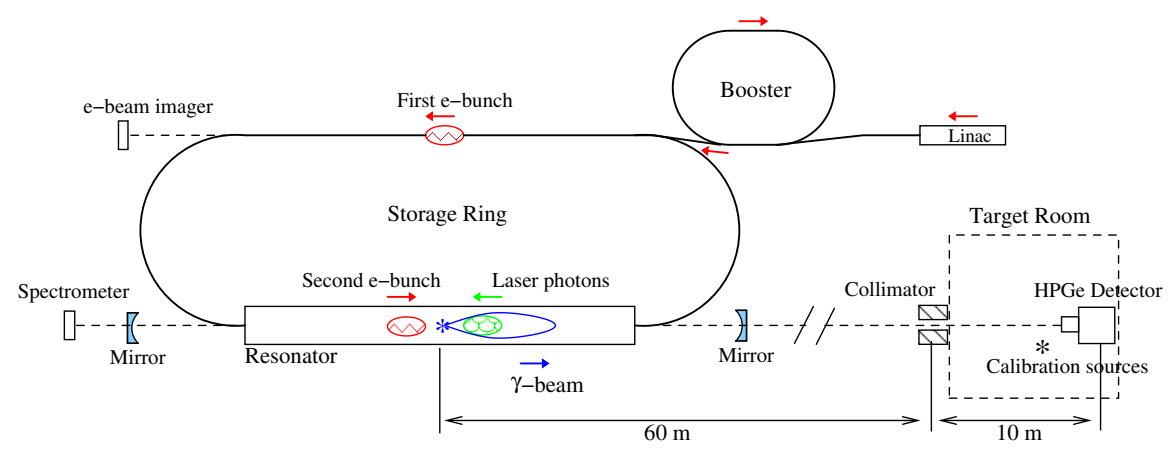

FIG. 7. (Color) Schematic of the $\mathrm{HI} \gamma \mathrm{S}$ beam production and measurement at Duke University.

the electron beam in the storage ring can also be adjusted by changing the field of the dipole magnets. The electron beam, consisting of two bunches separated by a half of the storage ring circumference, is used to drive the FEL. The FEL photons from the first (second) electron bunch collide with electrons in the second (first) bunch. The resultant high intensity gamma beam is transported in vacuum to a target room after passing through a lead collimator placed 60 meters downstream of the collision point and in front of the target room.

The energy spectrum of a $\mathrm{HI} \gamma \mathrm{S}$ beam is measured by a large volume $123 \%$ efficiency HPGe detector installed at the end of the target room 10 meters downstream from the collimator. The gamma-ray radiation sources of ${ }^{226} \mathrm{Ra}$ and ${ }^{60} \mathrm{Co}$ as well as the nature background from ${ }^{40} \mathrm{~K}$ are used for the detector energy calibration.

The FEL lasing spectrum is measured by a spectrometer, and the electron beam emittance is monitored by a synchrotron radiation profile monitor.

\section{B. Measurements with a large collimation aperture}

In the first experiments, a $\mathrm{HI} \gamma \mathrm{S}$ beam collimated by a lead aperture with a radius of $12.7 \mathrm{~mm}(\alpha \approx 9)$ was used to determine the energy and energy spread of the electron beam in the Duke storage ring.

To demonstrate the capability and limitation of the Compton scattering technique, the Duke storage ring dipole field was slightly adjusted with an increment of
$0.02 \mathrm{MeV}$ in the sequence of $461.06 \mathrm{MeV} \rightarrow$ $461.08 \mathrm{MeV} \rightarrow 461.10 \mathrm{MeV} \rightarrow 461.12 \mathrm{MeV} \rightarrow 461.14 \mathrm{MeV}$ in terms of the set energy of the storage ring. Note that the actual electron beam energy was different from that of the set energy at few $10^{-3}$ level. However, without a substantial hysteresis effect, the beam energy could be changed in a small range with a relative accuracy of $10^{-5}$ as determined by the controllability of the set energy of the storage ring. Therefore, the actual energy of the electron beam was precisely adjusted by an increment of about $0.02 \mathrm{MeV}$ with an uncertainty of about $0.004 \mathrm{MeV}$.

At each set energy of the storage ring, the FEL wiggler setting was also slightly adjusted in order to keep the lasing wavelength constant. The FEL spectrum parameters (peak $\lambda_{\mathrm{ph}}$ and linewidth $\sigma_{\lambda_{\mathrm{ph}}}$ ) measured by the spectrometer after adjustments are summarized in Table I. The electron beam emittances were measured by the synchrotron radiation profile monitor. The measured horizontal emittance is about $10 \mathrm{~nm} \mathrm{rad}$. The measurement of the vertical emittance is limited by diffraction effects, and the estimated vertical emittance is less than $1 \mathrm{~nm}$ rad. This assures that the influence of the vertical emittance on the gamma beam spectrum [Eq. (16)] can be neglected.

For each set energy of the storage ring, the energy spectrum of the $\mathrm{HI} \gamma \mathrm{S}$ beam was collected for about 20 min. A typical measured spectrum with the simultaneously recorded gamma-ray calibration source peaks are shown in Fig. 8. The energy calibration curve of the HPGe

TABLE I. Comparison of the electron beam energy and energy spread determined using both Eq. (16) and Eq. (12) for a collimation aperture of $12.7 \mathrm{~mm}$ radius. The uncertainty shown in the table represents the overall uncertainty of the measurement.

\begin{tabular}{lcccccc}
\hline \hline $\begin{array}{l}\text { Set energy } \\
(\mathrm{MeV})\end{array}$ & \multicolumn{2}{c}{ FEL wavelength $^{\mathrm{a}}$} & \multicolumn{2}{c}{$E$-beam energy $E_{e}(\mathrm{MeV})$} & \multicolumn{2}{c}{$E$-beam spread $\sigma_{E_{e}} / E_{e}\left(\times 10^{-4}\right)$} \\
\hline 461.06 & $791.260 \pm 0.032$ & 0.812 & $459.063 \pm 0.013$ & $459.066 \pm 0.013$ & $6.5 \pm 0.5$ & $6.6 \pm 0.5$ \\
461.08 & $791.250 \pm 0.032$ & 0.826 & $459.084 \pm 0.013$ & $459.089 \pm 0.013$ & $6.6 \pm 0.5$ & $6.3 \pm 0.5$ \\
461.10 & $791.218 \pm 0.032$ & 0.836 & $459.098 \pm 0.013$ & $459.105 \pm 0.013$ & $6.4 \pm 0.5$ & $6.3 \pm 0.5$ \\
461.12 & $791.210 \pm 0.032$ & 0.860 & $459.115 \pm 0.013$ & $459.128 \pm 0.013$ & $6.1 \pm 0.5$ & $6.0 \pm 0.5$ \\
461.14 & $791.184 \pm 0.032$ & 0.888 & $459.135 \pm 0.013$ & $459.146 \pm 0.013$ & $6.3 \pm 0.5$ & $6.3 \pm 0.5$ \\
\hline \hline
\end{tabular}

${ }^{\mathrm{a}}$ The FEL peak wavelength $\lambda_{\mathrm{ph}}$ and rms linewidth $\sigma_{\lambda_{\mathrm{ph}}}$ are determined by fitting a Gaussian function on the measured lasing spectrum. 


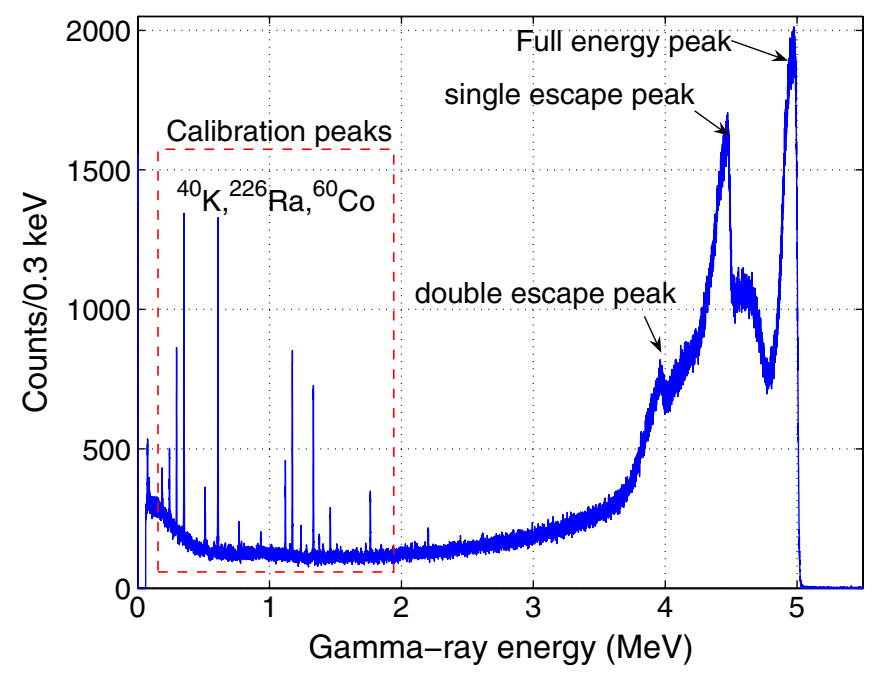

FIG. 8. (Color) A typical $\mathrm{HI} \gamma \mathrm{S}$ beam spectrum measured by a large volume $123 \%$ efficiency HPGe detector. The radiation sources of ${ }^{226} \mathrm{Ra}$ and ${ }^{60} \mathrm{Co}$ as well as the nature background from ${ }^{40} \mathrm{~K}$ are used in the real time for the detector energy calibration.

detector is shown in Fig. 9 to illustrate the energy linearity of the detector.

The high energy edges of the measured spectra for different storage ring set energies are shown in Fig. 10. We can see the gamma beam spectrum edge shifts to a higher energy accordingly as the storage ring set energy is increased from 461.06 to $461.14 \mathrm{MeV}$ with increments of $0.02 \mathrm{MeV}$ per step. The electron beam energy and energy spread fitted from these edges are summarized in Table I. The least squares fitting method has been used to fit Eq. (16), and a typical fitting result is illustrated in Fig. 11.

The accuracy of the electron beam energy measurement is mainly affected by the uncertainties in the determina-

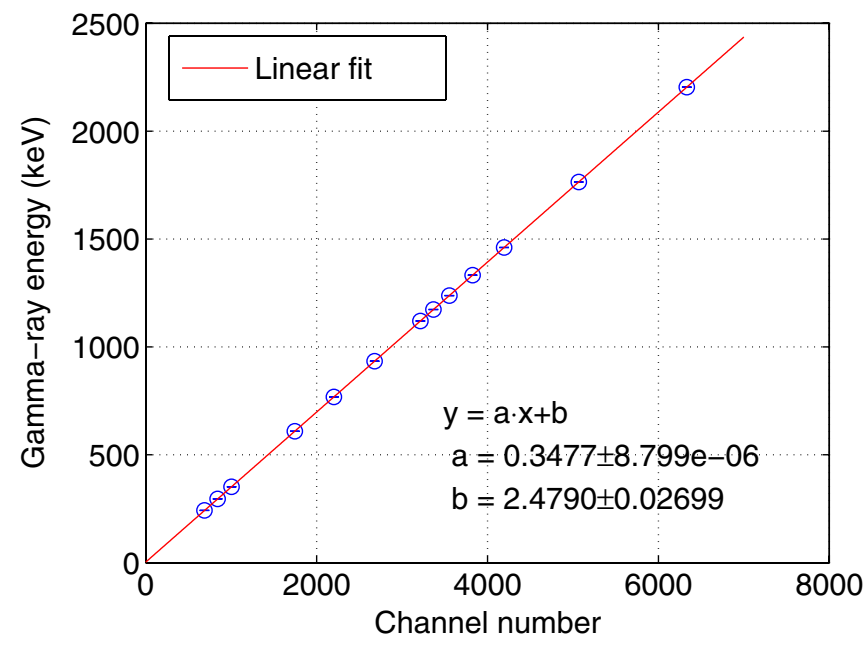

FIG. 9. (Color) The calibration curve of the HPGe detector. The straight line is a linear fit of the peak energies of the calibration sources.

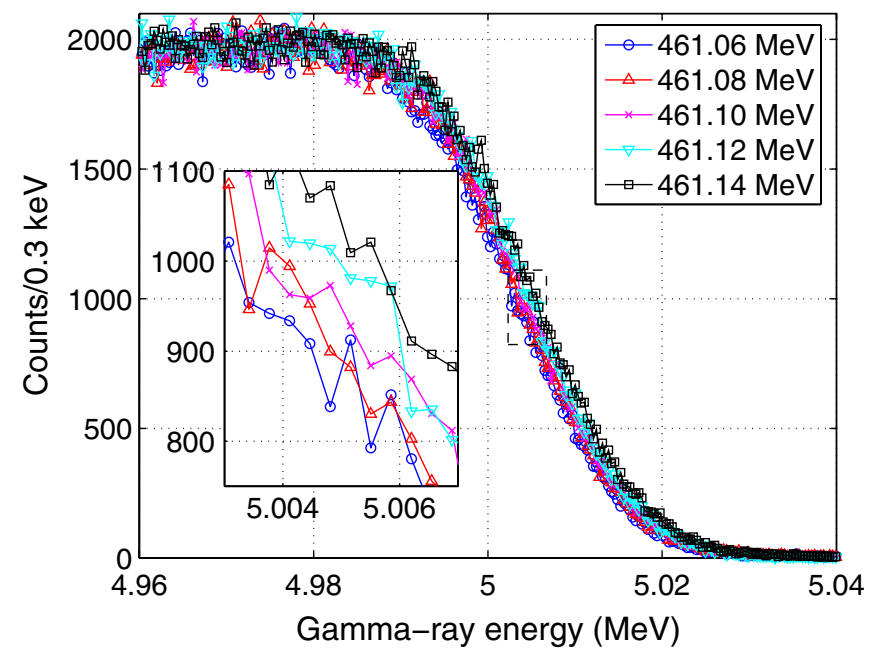

FIG. 10. (Color) High energy edges of the measured $\mathrm{HI} \gamma \mathrm{S}$ beam spectra for different storage ring set energy which is increased from 461.06 to $461.14 \mathrm{MeV}$ with increments of $0.02 \mathrm{MeV}$ per step. The inset is the magnified plot around the gamma-ray energy of $5.005 \mathrm{MeV}$.

tions of the gamma beam spectrum edge as well as the FEL peak wavelength. These uncertainties can be further divided into two types: systematic errors and statistical errors. The systematic errors arise from the calibration of the HPGe detector and the spectrometer, while the statistical errors arise from the intensity fluctuations in the measured gamma beam spectrum and the measured FEL spectrum. For example, the contributions of these individual errors to the uncertainty of the electron beam energy measurement are summarized in Table II for the measurement at the

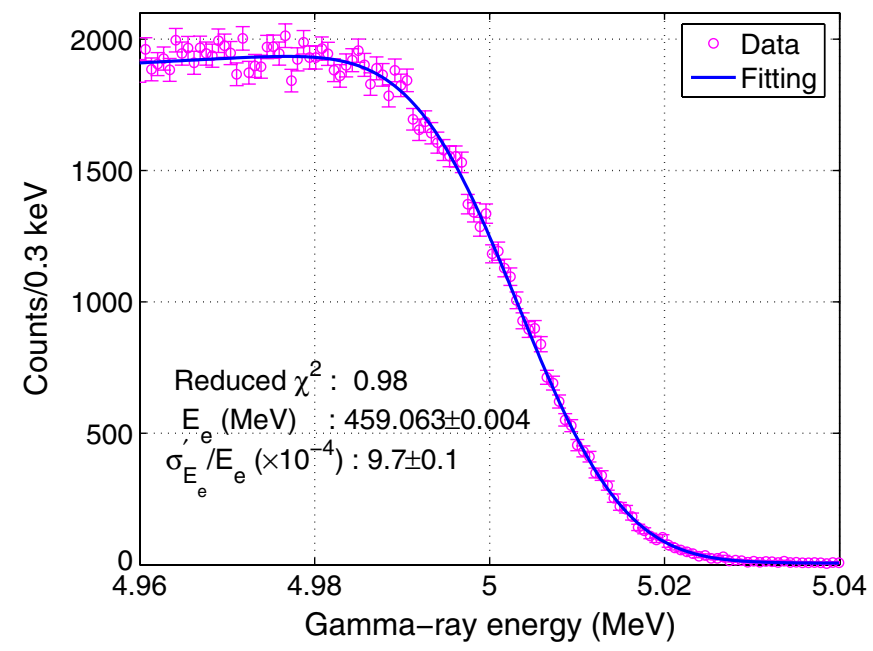

FIG. 11. (Color) An illustration of the fitting on the high energy edge of the measured gamma beam spectrum. The least squares method is used to fit Eq. (16). The goodness of fit is given by the reduced $\chi^{2}$. The fit electron beam energy $E_{e}$ and relative energy spread $\sigma_{E_{e}}^{\prime} / E_{e}$ as well as the fitting errors associated with them are also shown in the plot. 
TABLE II. Uncertainty of the electron beam energy measurement at the storage ring set energy of $461.06 \mathrm{MeV}$.

\begin{tabular}{|c|c|c|c|c|c|c|}
\hline \multirow[t]{2}{*}{ Error types } & \multicolumn{3}{|c|}{ Gamma beam } & \multicolumn{3}{|c|}{ FEL } \\
\hline & $\delta E_{\gamma}(\mathrm{keV})$ & $\delta E_{e}^{i}(\mathrm{MeV})^{\mathrm{a}}$ & $\delta E_{e}^{i} / E_{e}\left(\times 10^{-5}\right)$ & $\delta \lambda_{\mathrm{ph}}(\mathrm{nm})$ & $\delta E_{e}^{i}(\mathrm{MeV})^{\mathrm{b}}$ & $\delta E_{e}^{i} / E_{e}\left(\times 10^{-5}\right)$ \\
\hline Statistical & 0.087 & 0.0040 & 0.87 & 0.0018 & 0.00052 & 0.11 \\
\hline Systematic & 0.188 & 0.0087 & 1.9 & 0.032 & 0.0092 & 2.0 \\
\hline
\end{tabular}

${ }^{\mathrm{a}}$ Contribution of the gamma beam measurement error $\delta E_{\gamma}$ to the uncertainty of the electron beam energy measurement $\delta E_{e}^{i}$, which is given by the formula $\delta E_{e}^{i} \approx 0.5\left(\delta E_{\gamma} / E_{\gamma}\right) E_{e}$.

${ }^{6}$ Contribution of the FEL spectrum peak error $\delta \lambda_{\mathrm{ph}}$ to the uncertainty of the electron beam energy measurement $\delta E_{e}^{i}$, which is given by the formula $\delta E_{e}^{i} \approx 0.5\left(\delta \lambda_{\mathrm{ph}} / \lambda_{\mathrm{ph}}\right) E_{e}$.

storage ring set energy of $461.06 \mathrm{MeV}$. The overall uncertainty $\delta E_{e}(68 \%$ confidence level) of the electron beam energy measurement is given by the square root of the quadratic sum of the individual uncertainty contribution $\delta E_{e}^{i}$, i.e., $\delta E_{e}=\sqrt{\Sigma_{i}\left(\delta E_{e}^{i}\right)^{2}}=0.013 \mathrm{MeV}$. Clearly, the systematic errors which arise from the calibrations of the HPGe detector and the spectrometer dominate the uncertainty of the electron beam energy measurement. For the measurement at the storage ring set energy $461.06 \mathrm{MeV}$, the overall relative uncertainty of $3 \times 10^{-5}$ was achieved, including both systematic and statistical errors. Similar accuracy is also achieved for all measured electron beam energies as summarized in Table I.

The accuracy of the electron beam energy measurement can also be affected by the alignment of the collimator to the gamma beam as well as the alignment of the FEL beam to the electron beam. Before the measurements, the collimator has been well aligned using a gamma-ray beam imaging system recently developed at Duke, which has a sub-mm resolution. This assures that the influence of the collimator misalignment on the accuracy of electron beam energy measurement can be neglected. At $\mathrm{HI} \gamma \mathrm{S}$, the Compton gamma-ray beam is produced inside a 54 meter long FEL resonator cavity. In order to achieve the FEL lasing, the electron beam and the photon beam must be well aligned, and the misalignment angle $\theta$ is less than $4 \times$ $10^{-4} \mathrm{mrad}$, which produces a close-to-ideal head-on collision configuration for Compton scattering. The relative uncertainty of the electron beam energy due to the misalignment angle $\theta$ can be approximated as $\theta^{2} / 4$ [3], which gives the relative uncertainty of about $10^{-7}$ to the electron beam energy measurement.

The determined electron beam energy versus the set energy of the storage ring is plotted in Fig. 12. Note that all the spectra shown in Fig. 10 are calibrated using the same calibration data. To improve the calibration error, the average of the calibration peaks of all five radiation source spectra is used to determine the calibration energy. Thus, the electron beam energies fitted from the high energy edges of the gamma beam spectra are sharing the same calibration errors, i.e., the systematic errors. Therefore, only the statistical errors of the electron beam energy measurements are shown in Fig. 12, excluding the systematic errors. We can see that the small change of $0.02 \mathrm{MeV}$ (i.e., the relative change of $4 \times 10^{-5}$ ) of the electron beam energy can be clearly detected by the Compton scattering technique. This experiment demonstrates that the relative uncertainty of the electron beam energy measurement due to the statistical errors must be smaller than $4 \times 10^{-5}$, otherwise the small change $(0.02 \mathrm{MeV})$ of the electron beam energy would not have been detected.

Because of the finite resolution of the HPGe detector (approximately $5 \mathrm{keV}$ in rms value for $5 \mathrm{MeV}$ gamma-ray photons) and the finite linewidth of the FEL spectrum, the energy spectrum of the gamma-ray beam has been broadened by both the detector response and the lasing spectrum. However, for simplicity, these broadening effects are not taken into account in the fitting model of Eq. (16). Thus, the fitting of this model to the high energy edge of measured gamma beam spectrum only yields the effective electron beam energy spread which includes both the detector resolution and FEL linewidth effects. Therefore, in order to correctly estimate the actual electron beam

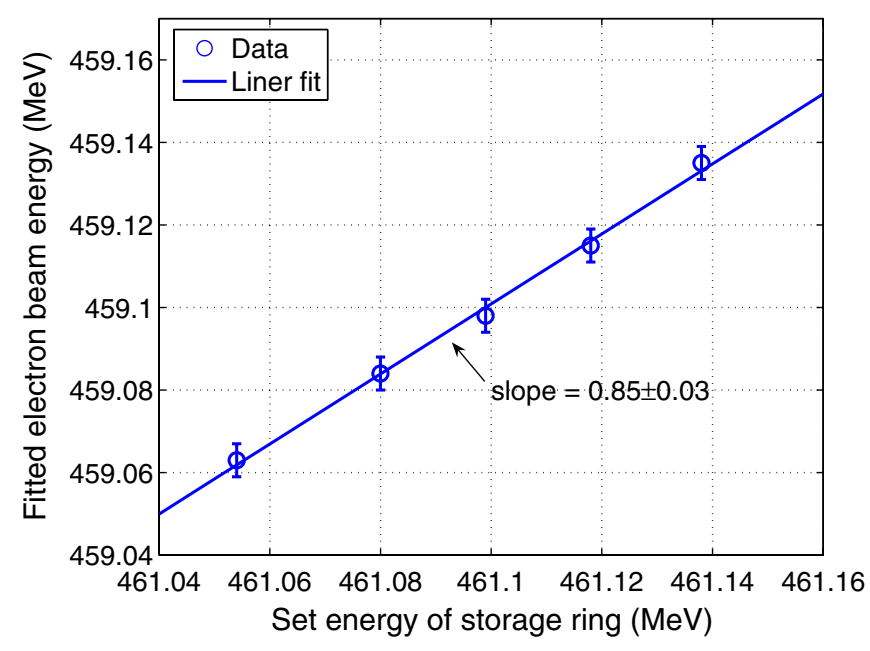

FIG. 12. (Color) Electron beam energy determined by Eq. (16) as a function of the set energy of the storage ring. The set energy has been corrected according to the digital-to-analog converter value which controls a power supply of dipole magnets. The vertical error bars only represent the statistical errors of the electron beam energy measurement, excluding the systematic errors. The straight line is the linear fit of the determined electron beam energies. The slope of the fit line as well as the fitting error associated with it are also shown in the plot. 
energy spread, these broadening effects must be removed if they are significant. This can be carried out using a simple formula

$$
\frac{\sigma_{E_{e}}}{E_{e}} \approx \sqrt{\left(\frac{\sigma_{E_{e}}^{\prime}}{E_{e}}\right)^{2}-\frac{1}{4}\left[\left(\frac{\sigma_{\mathrm{det}}}{E_{\gamma}}\right)^{2}+\left(\frac{\sigma_{\lambda_{\mathrm{ph}}}}{\lambda_{\mathrm{ph}}}\right)^{2}\right]},
$$

where $\sigma_{E_{e}}^{\prime}$ is the effective electron beam energy spread which is directly fit from the measured gamma beam spectrum using Eq. (16), $\sigma_{\text {det }}$ is the energy resolution of the detector, and $\sigma_{\lambda_{\mathrm{ph}}}$ is the linewidth of the FEL spectrum. The electron beam energy spread $\sigma_{E_{e}} / E_{e}$ shown in Table I has been corrected using this formula. The uncertainty of the energy spread measurement is estimated using the gamma spectrum fitting error and the errors of the detector resolution and lasing linewidth.

The electron beam energy and energy spread determined by Eq. (12) are also shown in Table I. Because of a large relative collimation factor $(\alpha \approx 9)$, Eq. (12) produces similar results to the ones produced by Eq. (16). The discrepancies between them are within the overall uncertainty of the measurement.

\section{Measurements with a small collimation aperture}

Many nuclear physics experiments require a more tightly collimated $\mathrm{HI} \gamma \mathrm{S}$ beam. Such a beam can be used to study the limitation of Eq. (12). With a collimation aperture of $6.35 \mathrm{~mm}$ radius, three energy measurements were conducted. The electron beam energy determined by both Eq. (16) and Eq. (12) are summarized in Table III. Because of a small relative collimation factor $(\alpha \approx 0.5)$, the electron beam energies determined by Eq. (12) are consistently higher than the results of Eq. (16) by as much as $0.7 \mathrm{MeV}$ or a relative difference of $1.5 \times 10^{-3}$. This agrees with the predication shown in Fig. 6 that for a tightly collimated gamma beam the electron beam energy could be overdetermined using Eq. (12). In this case, Eq. (12) cannot be applied to accurately determine the electron beam energy.

\section{DISCUSSIONS AND CONCLUSIONS}

In this work, the energy spectra of $\mathrm{HI} \gamma \mathrm{S}$ beams, measured with a large volume HPGe detector, have been used to determine the electron beam energy and energy spread.
This is acceptable when the full energy peak, the part of gamma spectrum corresponding to the total energy absorption, is clearly separated from the Compton background and single or double escape peaks. However, under certain circumstances, the full energy peak can be buried in the measured spectrum. This happens when the span of the higher energy edge of the gamma beam is comparable to or wider than the energy separation between the full energy peak and single escape peak. In this case, the gamma beam energy distribution needs to be reconstructed from the measured spectrum before being used for the determination of the electron beam energy. We have developed a novel end-to-end spectrum reconstruction method [22] to recover the gamma beam energy distribution even when the full energy peak is completely overwhelmed by the Compton background and escape peaks.

Equation (12) has been used to determine the electron beam energy in several published works $[3,4,8,9]$. However, this equation only takes into account the influence of the electron beam energy spread on the gamma beam spectrum. By ignoring other factors, this formula has a substantial limitation in its applications. We have demonstrated that it can produce inaccurate results for a well collimated gamma-ray beam with a relative collimation factor $\alpha \leq 4$.

According to Eq. (17), a small $\alpha$ can be the result of a low electron beam energy, a large electron beam energy spread, and a small angular divergence of a collimated gamma beam. Therefore, under certain beam conditions, for example, with a low energy storage ring, we need to open up the collimation aperture in order to apply Eq. (12). The advantage of opening up the collimation aperture for energy measurement of a low energy electron beam was also recognized in a recent publication [11]. However, this may not always be possible because the angular divergence of the gamma-ray beam can be limited by the angular acceptance of the gamma-ray beam transport line and the gamma-ray detector. For example, the maximum angular divergence of the gamma-ray beam at the $\mathrm{HI} \gamma \mathrm{S}$ facility is only about $0.5 \mathrm{mrad}$ which is limited by the angular acceptance of the vacuum chamber in a dipole magnet (vertical limit) and by the transport line (horizontal limits).

To overcome the limitations of Eq. (12), we have derived a new Eq. (16) to include the emittance and collimation effects. Using this equation, we have accurately deter-

TABLE III. Comparison of the electron beam energy determined by both Eq. (16) and Eq. (12) for a collimation aperture with a radius of $6.35 \mathrm{~mm}$.

\begin{tabular}{lccc}
\hline \hline $\begin{array}{l}\text { Set-energy } \\
(\mathrm{MeV})\end{array}$ & \multicolumn{2}{c}{$\begin{array}{c}\text {-beam energy } \\
E_{e}(\mathrm{MeV})\end{array}$} & $\begin{array}{c}\text { Discrepancy }(\mathrm{MeV}) \\
E_{e}^{12}-E_{e}^{16}\end{array}$ \\
\hline 463.00 & Eq. $(16)$ & Eq. $(12)$ & 0.72 \\
462.00 & $460.95 \pm 0.12$ & $461.67 \pm 0.12$ & 0.59 \\
461.00 & $460.19 \pm 0.13$ & $460.78 \pm 0.12$ & 0.51 \\
\hline \hline
\end{tabular}

${ }^{\mathrm{a}}$ The discrepancy of the determined electron beam energy between Eq. (12) and Eq. (16). 
mined the energy of an electron beam in the Duke storage ring with a relative uncertainty of $3 \times 10^{-5}$, including both systematic and statistical errors.

This level of energy measurement accuracy of a few $10^{-5}$ is comparable to that using the resonant spin depolarization technique. It has also been achieved using the Compton scattering technique in previous measurements [4,9] carried out for high energy storage rings above $1 \mathrm{GeV}$. This work reports the electron beam energy measurement with a similar accuracy of a few $10^{-5}$ for a low energy storage ring at a few hundred $\mathrm{MeV}$. In addition, we showed for the first time that a small energy change about $0.02 \mathrm{MeV}$ of a $460 \mathrm{MeV}$ electron beam (i.e., a relative change of $4 \times 10^{-5}$ ) by varying storage ring dipole field can be directly detected using the Compton scattering technique.

\section{ACKNOWLEDGMENTS}

We would like to thank the scientists and staff at the Duke Free Electron Laser Laboratory (DFELL) for their support on the machine operation. This work is supported by U.S. Department of Defense Medical FEL Program as administered by the AROSR under Contract No. FA955004-01-0086 and U.S. Department of Energy Grant No. DEFG05-91ER40665 (Y. K. Wu, C. Sun, and J. Li). This work is also supported by the U.S. Department of Energy under Grants No. DE-FG02-97ER41033 and No. DE-FG5206NA26155 (A. P. Tonchev and G. Rusev).

[1] D. P. Barber et al. (ARGUS Collaboration and Crystal Ball Collaboration), Phys. Lett. 135B, 498 (1984).

[2] V.E. Blinov et al., Nucl. Instrum. Methods Phys. Res., Sect. A 494, 81 (2002).

[3] R. Klein, T. Mayer, P. Kuske, R. Thornagel, and G. Ulm, Nucl. Instrum. Methods Phys. Res., Sect. A 384, 293 (1997).
[4] R. Klein, P. Kuske, R. Thornagel, G. Brandt, R. Grgen, and G. Ulm, Nucl. Instrum. Methods Phys. Res., Sect. A 486, 545 (2002).

[5] J. Schwinger, Phys. Rev. 75, 898 (1949).

[6] Y. Derbenev, A. Kondratenko, S. Serednyakov, A. Skrinsky, G. Tumaikin, and Y. Shatunov, Part. Accel. 10, 177 (1980).

[7] K. Chouffani, F. Harmon, D. Wells, J. Jones, and G. Lancaster, Phys. Rev. ST Accel. Beams 9, 050701 (2006).

[8] H. Ohgaki, H. Toyokawa, K. Kudo, N. Takeda, and T. Yamazaki, Nucl. Instrum. Methods Phys. Res., Sect. A 455, 54 (2000).

[9] N. Muchnoi, S. Nikitin, and V. Zhilich, in Proceedings of the European Particle Accelerator Conference (EPS-AG, Edinburgh, Scotland, 2006), pp. 1181-1183.

[10] I. C. Hsu, C.-C. Chu, and C.-I. Yu, Phys. Rev. E 54, 5657 (1996).

[11] R. Klein et al., Phys. Rev. ST Accel. Beams 11, 110701 (2008).

[12] V. N. Litvinenko and J. M. J. Madey, Proc. SPIE Int. Soc. Opt. Eng. 2521, 55 (1995).

[13] V.B. Berestetskii, E. M. Lifshitz, and L.P. Pitaevskii, Quantum Electrodynamics (Butterworth-Heinemann, Oxford, 1982), 2nd ed.

[14] V. N. Litvinenko et al., Phys. Rev. Lett. 78, 4569 (1997).

[15] A. M. Sandorfi, J. LeVine, C. E. Thorn, G. Giordano, and G. Matone, IEEE Trans. Nucl. Sci. 30, 3083 (1983).

[16] F. R. Arutyunian and V. A. Tumanian, Phys. Lett. 4, 176 (1963).

[17] S. H. Park, Ph.D. thesis, Duke University, 2000.

[18] C. Sun and Y. K. Wu (to be published).

[19] K. Yokoya, User Manual of CAIN, version 2.35 (2003).

[20] Y. K. Wu, N. A. Vinokurov, S. Mikhailov, J. Li, and V. Popov, Phys. Rev. Lett. 96, 224801 (2006).

[21] Y.K. Wu, in Proceedings of Particle Accelerator Conference (IEEE, Albuquerque, NM, 2007), pp. 12151217.

[22] C. Sun, Y.K. Wu, G. Rusev, and A.P. Tonchev, Nucl. Instrum. Methods Phys. Res., Sect. A 605, 312 (2009). 